\title{
CT-based Assessment of Myocardial Bridging in Patients with Acute Chest Pain and No Atherosclerotic Etiology
}

\author{
Alexandra Stănescu' ${ }^{1}$, Zsuzsanna Suciu² ${ }^{2}$ Nora Rat ${ }^{2}$, Mirabela Morariu $^{2}$, Monica Chițu ${ }^{2}$ \\ ${ }^{1}$ Center of Advanced Research in Multimodality Cardiac Imaging, Cardio Med Medical Center, Tîrgu Mureș, Romania \\ ${ }^{2}$ Clinic of Cardiology, University of Medicine and Pharmacy, Tîrgu Mureș, Romania
}

\section{ABSTRACT}

Introduction: The term "myocardial bridging" is used to describe an anatomic variant where a band of cardiac muscle overlies a segment of an epicardial coronary artery. It is a highly debated topic, because it can cause conditions such as acute coronary syndrome. Myocardial bridging (MB) can be diagnosed using invasive procedures, but also non-invasive ones, such as Multislice Computed Tomography Angiography (MSCTA). Objectives: A comparative analysis was performed on the patients who were admitted to the clinic with typical angina, ischemic ECG changes and muscular bridging shown on MSCTA, and patients with the same symptoms, but without MB. A sub-study was also undertaken in which the MB site and ischemia revealed by thickening of the myocardial muscle, using 3D Polar Mapping, were compared. Materials and methods: A retrospective study assessed 59 patients with typical angina pectoris, shortness of breath and clinical appearance of an acute coronary syndrome, and for whom MSCTA was carried out. Patients were divided into two groups: Group 1 - patients with MB, and Group 2 - patients without MB. Thirty patients in Group 1 had 3D polar mapping to evaluate the thickness of the myocardial muscle. Results: The mean age of our patients with muscular bridging was $55.51 \pm 11.4$ years, CI $51.57-59.45$ years. Patients without MB had a mean age of $59.17 \pm 9.6$ years, CI $54.98-63.6$ years, $p=0.211 .24 .32 \%$ of the patients with $\mathrm{MB}$ were females and $60.86 \%$ from the patients without $\mathrm{MB}$ were males, $\mathrm{p}=$ $0.040 .40 .54 \%$ of patients presented with $\mathrm{MB}$ in the first segment of the LAD and $15.62 \%$ had an MB in the second segment of LAD. In patients with an ischemic site smaller than $2 \mathrm{~cm}$ of the $\mathrm{MB}$, the ischemic myocardial area was more pronounced compared to the patients with higher length $\mathrm{MB}(21.85 \pm 6.123 \%$ vs. $17.62 \pm 5.856 \%)$. Conclusions: MSCTA is an important procedure that contributes to the clinical investigations of patients with typical angina and suspected acute coronary syndrome. There is a good positive correlation between the location of the MB and the ischemic segments as shown on 3D CT-based polar maps.

Keywords: 3D polar mapping, myocardial bridging, angina pectoris

\section{ARTICLE HISTORY}

Received: 10 March, 2016

Accepted: 13 May, 2016

\section{CORRESPONDENCE}

Nora Rat

Str. Gheorghe Marinescu nr. 38 540139 Tîrgu Mureș, Romania.

Tel: +40 265215551

E-mail: ratnora@gmail.com 


\section{INTRODUCTION}

Myocardial bridging (MB) is defined as a congenital coronary anomaly, ${ }_{1}^{1}$ represented by a segment of a major epicardial coronary artery that proceeds intramurally through the myocardium, beneath the muscle bridge. MB can cause variable degrees of systolic compression. It was first described in 1737 by Reyman during an autopsy. ${ }^{2}$ Portman and Iwing reported the first case of MB detected by tomography coronary angiography. ${ }^{3}$ Because it is a "silent" condition which may be associated with severe ischemia caused by the systolic compression of the coronary artery, MB is a subject frequently debated in the literature. The prevalence of $\mathrm{MB}$ is still unknown, although from autopsy studies its reported as being present in 25$85 \%$ of the patients. ${ }^{4}$ Diagnostic imaging methods such as angiography suggest that the incidence of $\mathrm{MB}$ ranges from $0.8 \%$ to $4.9 \% .{ }^{5}$ Non-invasive procedures tend to be more frequently used by clinicians in diagnosing the etiology of coronary artery disease, due to their high specificity. Moreover, many patients accept these procedures as they are not invasive and can be performed ambulatory. ${ }^{6}$ Multislice Computed Tomography Angiography (MSCTA) is the primary non-invasive imaging technique used in clinical practice for the diagnosis of MB.

The present study aimed to compare the clinical characteristics of patients presenting with typical angina pectoris and unconfirmed suspicion of an acute coronary syndrome and MSCTA-revealed MB, with patients presenting in the emergency room with unconfirmed ACS and MSCTA-non-revealed MB.7,8

Our additional aim was to investigate the functional significance of MB as expressed by the patients' hemodynamics, using MSCTA-based 3D reconstructions of the left ventricular cavities.

\section{MATERIALS AND METHODS}

Fifty-nine patients presenting at the emergency department from January 2015 - September 2015 for typical angina and an ECG diagnosis of myocardial ischemia who were subsequently referred for 64-Slice Computed Tomography Angiography were enrolled in the study. None of the patients underwent percutaneous coronary angioplasty at that visit, nor presented a history of coronary angioplasty. All patients were subjected to blood sampling and MSCTA. According to the radiological protocol for lowering the heart rate, Metoprolol was administered to maintain the heart rate at under sixty beats per minute. An intravenous line was inserted into the right antecubi- tal vein for contrast administration. ${ }^{9,10}$ Data from the patients' files were collected, and all the data were entered into an electronic database. Patients were divided into two groups: 36 patients with muscular bridging demonstrated by MSCTA (Group 1) and 23 patients without muscular bridging (Group 2). The following parameters were analyzed: Ca Scoring, serum biomarkers, demographic and clinical characteristics, MSCTA biomarkers.

The study has been carried out in accordance with the code of ethics of the World Medical Association's Declaration of Helsinki. All patients gave written informed consent, and the study protocol was approved by the ethics committee of the Cardio Med Medical Center, the center where the study was conducted.

\section{SUB-STUDY FOR CT ANALYSIS OF MB}

In the CT analysis sub-study 30 patients with muscular bridging on whom MSCTA reconstructions of the ventricular cavities had been carried out, were enrolled. This non-invasive procedure consisted in the measurement of the thickness of the ventricular walls and the detection of thinned areas, which showed ischemia and contractility dysfunction. The left ventricle was divided into seventeen segments for regional analysis of left ventricular function or myocardial perfusion according to AHA (Figure 1). ${ }^{9}$ The area with contractility dysfunction is shown

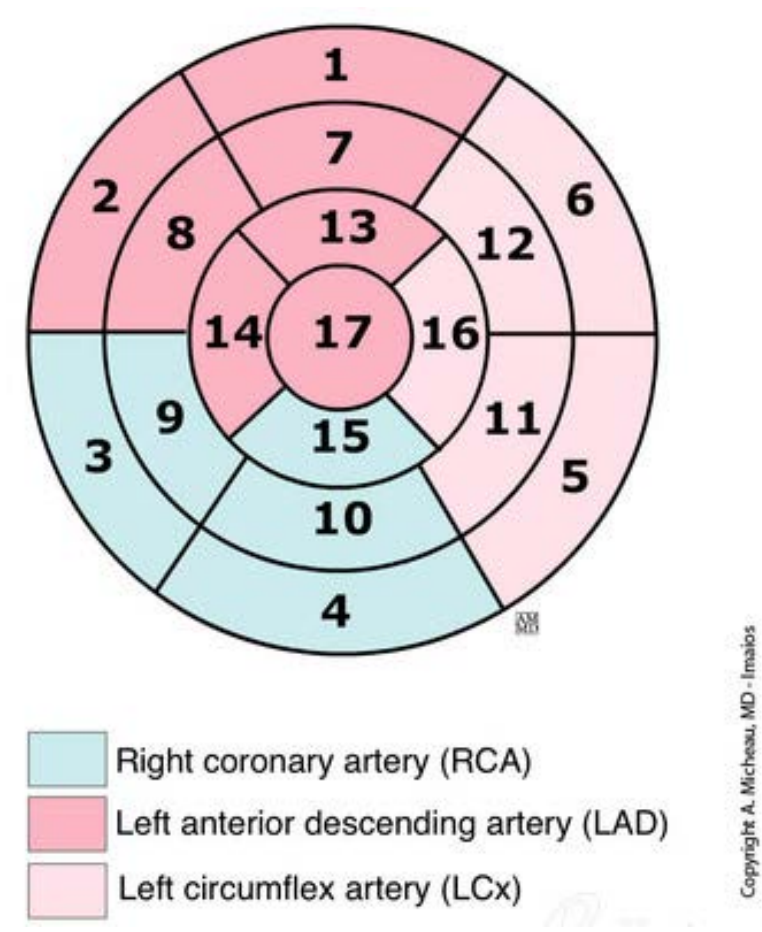

FIGURE 1. Left ventricle segmentation 


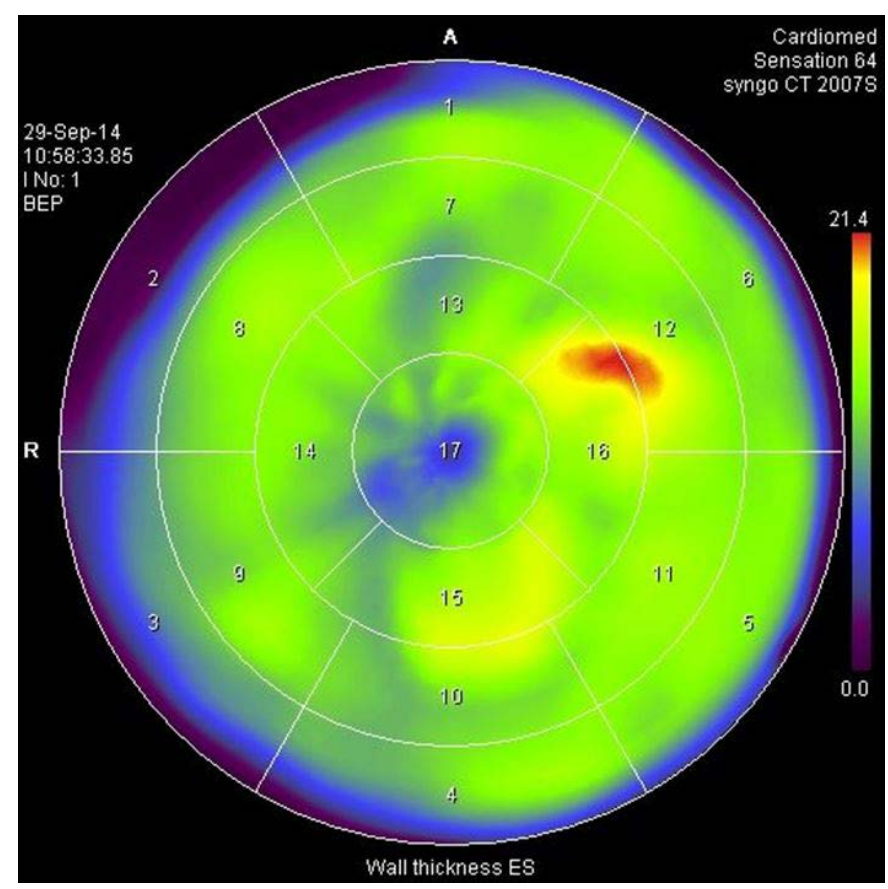

FIGURE 2. Polar map showing a thickening of the anterior wall colored in purple-blue

colored in a blue-purple spot on both the polar map and the 3D image of the ventricle (Figure 2). The thickness of the left ventricular wall was measured at end-systolic and end-diastolic phases, resulting in a polar map of the

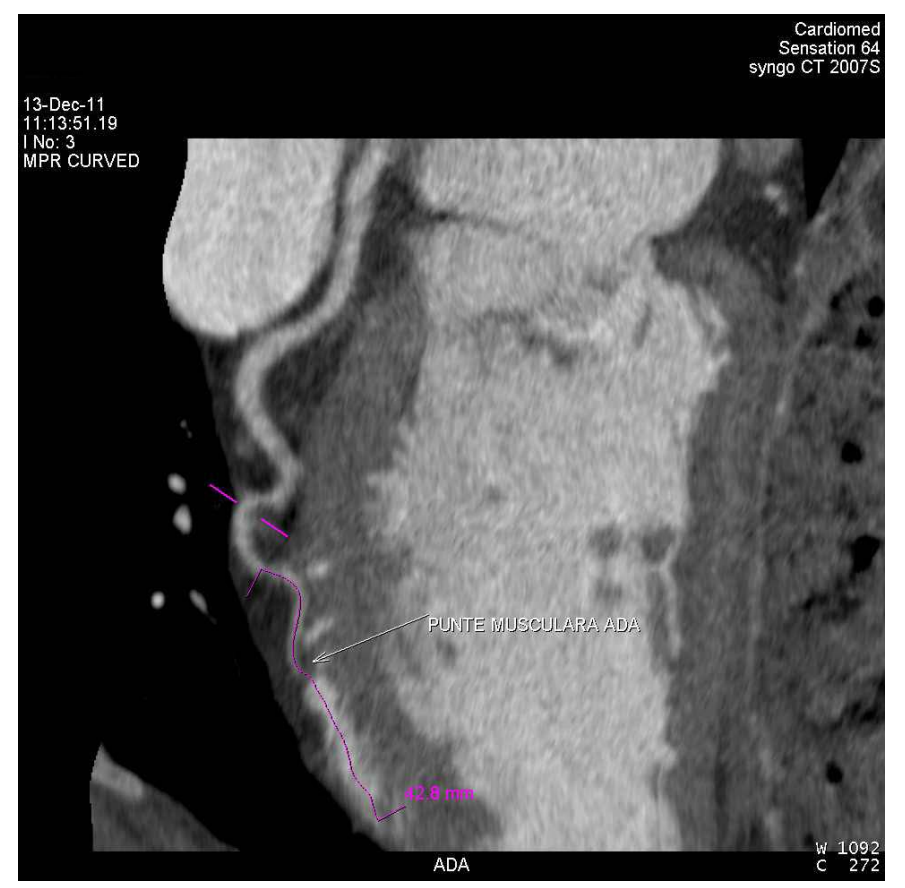

FIGURE 4. Myocardial thickening (blue spot on the left image) shown on polar map; 3D reconstruction of the left ventricle during systole (on the right)

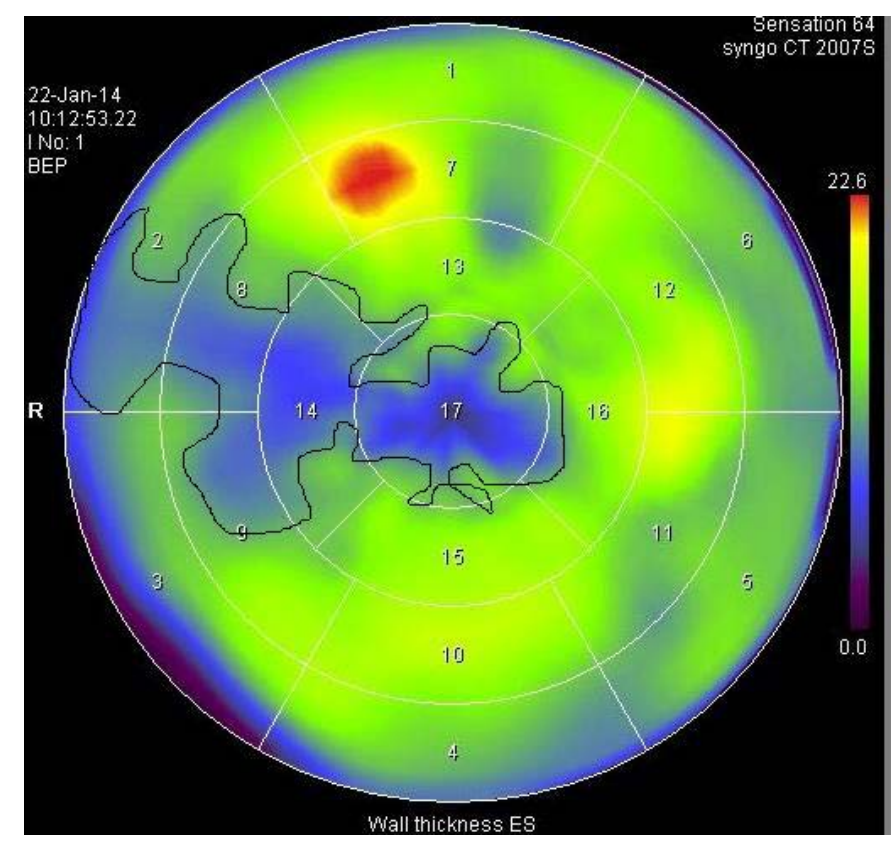

FIGURE 3. Polar map showing an ischemic zone marked with blue color

left ventricle showing all the segments colored according to their thickness or systolic thickening (Figures 3 and 4).

\section{RESULTS}

The mean age of patients with MB was $55.51 \pm 11.4$ years, CI 51.57-59.45 years; patients without MB had a mean age of $59.17 \pm 9.6$ years, CI $54.98-63.6$ years.

$24.32 \%$ of patients with MB were females, while $60.86 \%$ without MB were males $(p=0.04)$.

The mean diastolic blood pressure was significantly higher in patients with $\mathrm{MB}(78.72 \pm 9.5 \mathrm{mmHg}$, CI 75.0581.79) compared to patients without MB $(72.08 \pm 11.68$ $\mathrm{mmHg}$, CI 67.03-77.14), $\mathrm{p}=0.029$.

Patients with MB presented significantly lower levels of cholesterol and triglycerides as compared to patients with chest pain associated with other etiologies. The mean level of cholesterol was $152.11 \pm 29.7 \mathrm{mg} / \mathrm{dl}$ (CI 129.28-174.94) in patients from Group 1, and $190.130 \pm 33.99 \mathrm{mg} / \mathrm{dl}$ (CI 175.43-204.83) in patients from Group2 $(\mathrm{p}=0.04)$. Also, patients in Group 1 presented a significantly higher mean triglycerides level of $94.3 \pm 19.83 \mathrm{mg} / \mathrm{dl}$ compared to patients in Group 2 (127.34 $\pm 40.84 \mathrm{mg} / \mathrm{dl}$, CI 109.69-145.01, $\mathrm{p}=0.037$ ).

Patients from Group 1 presented anterior ECG ischemic changes in $50 \%$ of the cases and $56.52 \%$ of the patients from the Group 2 had anterior ECG ischemic changes, OR: 1.3. $(\mathrm{p}=0.8)$. 
TABLE 1. Quantification of myocardial ischemia caused by MB

\begin{tabular}{lccc}
\hline Parameter & $\begin{array}{c}\text { ADA } \\
\mathbf{n = 2 9}\end{array}$ & $\begin{array}{c}\text { Non-ADA } \\
\mathbf{n = 7}\end{array}$ & p value \\
& & & 0.01 \\
$\begin{array}{l}\text { Decreased contraction amplitude (\%) } \\
\quad \text { Mean } \pm \text { SD }\end{array}$ & $23.75 \pm 11.140$ & $11.83 \pm 1.169$ & \\
$\quad$ 95\% confidence interval & $19.04-28.455$ & $10.606-13.060$ & \\
Length of MB (cm) & $2.447 \pm 1.105$ & $2.566 \pm 0.383$ & \\
$\quad$ Mean \pm SD & $1.980-2.914$ & $2.165-2.969$ & \\
$\quad 95 \%$ confidence interval & & & 0.0006 \\
Area of the myocardial ischemia (\%) & $18.625 \pm 4.412$ & $11.5 \pm 1.049$ & \\
$\quad$ Mean \pm SD & $16.762-20.488$ & $10.399-12.601$ & \\
$\quad 95 \%$ confidence interval & 6 & 2 & 0.05 \\
The depth of MB (mm) & & & \\
\hline
\end{tabular}

\section{CT SUBSTUDY RESULTS}

CT characteristics of the population enrolled in the substudy are presented in Table 1.

The mean length of the muscular bridging was $2.194 \pm$ $0.914 \mathrm{~cm}$.

$40.54 \%$ of patients presented with MB in the first segment of the $\mathrm{LAD}$ and $15.62 \%$ had $\mathrm{MB}$ at the second segment of LAD. In patients with an MB length smaller than two $\mathrm{cm}$, the ischemic myocardial area was more pronounced compared to the patients with an MB length greater than two $\mathrm{cm}(21.85 \pm 6.123 \%$ vs. $17.62 \pm 5.856 \%)$. In $70.45 \%$ of the cases, patients presented a surface MB, compared to 29.55\% who had a deep MB.

Ca Scoring mean values were $90.63 \pm 203.69$ (CI 40.56180.56), with no significant differences between the groups (Table 2).

\section{DISCUSSIONS}

In this study, the presence of muscular bridging was much higher in male patients. Patients with MB had a lower level of cholesterol and triglycerides, compared with patients with no MB. ${ }^{11}$ This is explained by the possible ischemic etiology of the chest pain in Group 2 patients, the association between coronary artery disease and high cholesterol

TABLE 2. Calcium Scoring values

\begin{tabular}{lccc}
\hline & MB group & $\begin{array}{c}\text { Non-MB } \\
\text { group }\end{array}$ & p value \\
\hline Ca Scoring $=0$ & $27 \%$ & $30 \%$ & 0.8 \\
Ca Scoring $<100$ & $56 \%$ & $53 \%$ & 0.9 \\
Ca Scoring $100-400$ & $10 \%$ & $14 \%$ & 0.1 \\
Ca scoring $>400$ & $7 \%$ & $3 \%$ & 0.08 \\
\hline
\end{tabular}

levels being well-known. In both groups, patients had anterior ECG changes, but without any significant difference between the groups. A higher percentage of muscular bridging was found in the second segment of the left anterior descending artery (LAD) compared to other locations. This could be explained by the trajectory of the LAD, which is distributed to the ventricular anterior wall, the part of the heart with the highest content of muscular fibers. The lack of association between Calcium Scoring and MB could be due to the low number of patients enrolled in the study, and this correlation should be further investigated. MSCTA represents an important procedure that contributes to the clinical investigation of patients with typical angina and a suspected acute coronary syndrome. ${ }^{12,13}$

\section{CONCLUSIONS}

There is a good positive correlation between the location of the $\mathrm{MB}$ and the ischemic segments showing on polar maps as thinned areas colored in blue, purple spots. Also, the 3D Polar Mapping procedure can be useful for identification of a correlation between the location of ischemic segments and the ECG location of ischemia.

\section{CONFLICT OF INTEREST}

None declared.

\section{ACKNOLEDGEMENT}

This research was supported by the Cardio Med Medical Center, project no. CAM/2015/RD/45.

\section{REFERENCES}

1. Erbel R, Ge J, Möhlenkamp S, et al. Myocardial Bridging - A Congenital Variant as an Anatomic Risk Factor for Myocardial 
Infarction? Circulation. 2009;120:357-359. DOI: 10.1161/ CIRCULATIONAHA.109.881367.

2. Reyman HC. Disertatio de vasis cordis propriis. Med Diss Univ Göttingen.7th Sept 1737;1-32.

3. PORSTMANN W, IWIG J Intramural coronary vessels in the angiogram. Fortschr Geb Rontgenstr Nuklearmed. 1960;92:129-133.

4. Bourassa MG, Butnaru A, Lesperance J, Tardif JC. Symptomatic myocardial bridges: overview of ischemic mechanisms and current diagnostic and treatment strategies. J Am Coll Cardiol. 2003;41:351-359. DOI: 10.1016/S0735-1097(02)02768-7.

5. Alegria JR, Herrmann J, Holmes DR Jr, Lerman A, Rihal CS. Myocardial bridging. Eur Heart J. 2005;26:1159-1168.

6. Nieman K, Oudkerk $M$, Rensing BJ, et al. Coronary angiography with multi-slice computed tomography. Lancet. 2001;357:599-603.

7. Mohamed Abdou. Myocardial bridging causing ischemia and recurrent chest pain: a case report. Int Arch Med. 2011;4:24. DOI: $10.1186 / 1755-7682-4-24$

8. Bayrak F, Degertekin M, Eroglu E, et al. Evaluation of myocardial bridges with 64-slice computed tomography coronary angiography. Acta Cardiol. 2009;64(3):341-346.

9. Schwarz ER, Klues HG, vom Dahl J, Klein I, Krebs W, Hanrath
P. Functional, angiographic and intracoronary Doppler flow characteristics in symptomatic patients with myocardial bridging: effect of short-term intravenous beta-blocker medication. J Am Coll Cardiol. 1996;27:1637-1645.

10. 10.Cerqueira MD, Weissman NJ, Dilsizian V, et al. Standardized Myocardial Segmentation and Nomenclature for Tomographic Imaging of the Heart. A Statement for Healthcare Professionals From the Cardiac Imaging Committee of the Council on Clinical Cardiology of the American Heart Association. Circulation. 2002;105:539-542.

11. Bourassa MG, Butnaru A, Lespérance J, Tardif JC. Symptomatic myocardial bridges: overview of ischemic mechanisms and current diagnostic and treatment strategies. J Am Coll Cardiol. 2003;41(3):351-359.

12. Hwang JH, Ko SM, Roh HG, et al. Myocardial Bridging of the Left Anterior Descending Coronary Artery: Depiction Rate and Morphologic Features by Dual-Source CT Coronary Angiography. Korean J Radiol. 2010;11:514-521. DOI: 10.3348/ kjr.2010.11.5.514

13. La Grutta L, Runza G, Lo Re G, et al. Prevalence of myocardial bridging and correlation with coronary atherosclerosis studied with 64-slice CT coronary angiography. Radiol Med. 2009;114(7):1024-1036. DOI: 10.1007/s11547-009-0446-y. 